\title{
Hybrid Power Systems Planning with Geographical Information System Models
}

\author{
P. J. Zorzano-Santamaría, A. Falces de Andrés, L. A. Fernández-Jiménez, E. García-Garrido, E. Zorzano-Alba, \\ M. Mendoza-Villena, P. Lara-Santillán, \\ Department of Electrical Engineering \\ E.T.S.I.I., University of La Rioja \\ Luis de Ulloa 20, 26004 Logroño, La Rioja (España) \\ Telef:+34 941 299477, fax:+34 941 299478, e-mail: pedrojose.zorzano@unirioja.es
}

\begin{abstract}
This paper presents planning models for hybrid distributed generation systems, as well as the results corresponding to a distribution system planning problem obtained using a new computational tool based on a Geographic Information System, GIS. This computational tool is a powerful instrument for analyzing energy resources and energy conversion technologies that can be used for the distribution networks expansion. It has been used in the economic evaluation of energy produced by hybrid systems. Thus, suitable models and techniques have initially been applied to obtain maps of solar and wind energy resources in a user defined area and maps of costs for hybrid systems, identifying the geographical locations that offer the best economic potential in distributed generation with renewable hybrid systems, supported (or not) with fuel to supply internal-combustion electric generators. Accurate evaluations of the cost of the produced energy need the use of geographic distributed costs corresponding to refuelling, installation and maintenance of the hybrid system.
\end{abstract}

The developed software tool is flexible, appropriate for studying different scenarios, and enables geographical analysis of the economic competitiveness of the hybrid systems with different distributed generation resources (photovoltaic, wind, hydraulic or biomass energy systems), and it is suitable to study the optimal expansion of existing power distribution networks (isolated hybrid systems versus connected ones).

\section{Key words}

Hybrid Power Systems, Optimal Planning, Distributed Generation (DG), Geographical Information Systems (GIS).

\section{Introduction}

This paper presents the computational models and the results obtained for the optimal location of electric hybrid generation equipment for distribution systems planning in areas with wind and solar or biomass resources. These models and results are related to power generation technologies that are also being subjects of worldwide current research interests [2], [3], [4], [5]; this paper focuses on the geographical relationships between the energy resources, their technological availability and electric power demand.

Thus, this paper describes the development of a set of planning tools and geographical databases on a GIS platform, for optimal distribution systems planning, integrating renewable and alternative energy resources (non-renewable), and related technologies taking into account the electric power infrastructures in the studied area (our case-study is La Rioja, Spain).

The model works with collected maps of regional renewable resources (available energy from wind and sun) or non-renewable energy resources. Other collected data in terms of geographical variability are economic data for evaluating fiscal incentives, taxes and duty exemptions and subsidies for equipment, installation, operation or maintenance and technological costs. Besides, the impact of produced and saved carbon dioxide (evaluated in terms of Kyoto Protocol compliance) can also be evaluated economically.

The resources, costs and electrical demand data have a strong geographical dependence; consequently, tools for geographical spatial analysis were required (using a Geographical Information System, GIS) in order to find good planning solutions. Recently the development of computational tools based on GIS platforms has also been used to integrate renewable energy resources into distribution systems planning [1].

In many published works, comparisons of different electricity supply systems are based on the evaluation of Levelized Energy Costs (LEC, €/kWh). Hence, better results can be achieved when different renewable resources are used in the same location, in order to obtain better global costs of common and shared equipment, installation, operation and maintenance of a hybrid power system, with different distributed generation 
technologies, and several demand scenarios. In each geographical location of study, the set of planning tools introduced in this paper enables a comparison of isolated or connected systems, taking into account the costs of the connection to the electric distribution networks, as well as different demand values for each consumption scenario.

\section{Computational Model}

In our model, the Levelized Energy Costs (LEC) of a hybrid distributed generation system corresponds to the cost of electricity supply ( $€ / \mathrm{kWh})$ per energy unit, for a combination of only three technologies, or subsystems (but it can be extended to other numbers). Those technologies can be implemented simultaneously in the same location: small wind turbines (W), solar photovoltaic panels (PV) and electric generating units with internal combustion engines $(G)$ that can be fed by diesel or bio-diesel oil. The model represents a suitable reliability of the hybrid system with this aggregation of distributed generation (DG) subsystems, using the power supply of the electric generating unit in the moments when the energy resources of other systems (wind, daylight) are not available or could not satisfy the power demand requirements.

\section{A. Required Data}

The initial data for each subsystem are selected from geographical energy resources data. In our example, wind resources for wind turbines, solar resources for photovoltaic panels and the availability (gas stations) and calorific properties of the fuel for the electric generating unit, and their performance for electric energy generation and emitted carbon dioxide rates, all over the geographic area to be studied.

The set of computational tools for distribution systems planning is extremely flexible, since hybrid systems are able to work isolated or connected to power distribution networks. Besides, it is possible to choose the percentage of every subsystem that can be used to meet demand (even to eliminate a subsystem, by choosing $0 \%$ utilization factor) and the annual hours of use of the electric generating unit.

Therefore, computational tools can be used to evaluate the LEC (in $€ / \mathrm{kWh}$ ) corresponding to the power supply by distributed energy resources, and comparing this cost with respect to those obtained from other electric power production technologies. These computational tools are integrated in a GIS platform described in [1] and its core is built by spatial analysis methodologies developed on GIS software [6].

\section{B. Modelling and Calculation Process}

1) Isolated hybrid distributed generation systems. The computational tools described in [1] determine the size $(\mathrm{PH})$ of the hybrid system in an isolated generation scenario to meet a specific demand (provided by the user) or to provide a certain installed power capacity $(Q$, in $\mathrm{kW}$, according to the user's specifications for PV, W and G). The hybrid system is resized according to the selected weight or utilization factors $(P V \%+W \%+G \%=1)$ of each hybrid subsystems, the energy resources to be used and the quantity of available resources in the studied area; this quantity is geographically distributed and obtained by suitable modules of the computational tools described in [1]. In this case, the Levelized Energy Costs, $L E C_{0}$, have been calculated for the characteristics of the selected hybrid system (photovoltaic and biodiesel electric generator).

For example, to determine the size (PHPV) of the photovoltaic subsystem of the hybrid system, first we calculate the power size from the input data.

The needed data to use are the following ones: $G_{A}$, Daily Annual Average Irradiation (in $\mathrm{W} \cdot \mathrm{h} / \mathrm{m}^{2}$.day);

$G_{0}$, Irradiation in Standard Conditions (STC) [7], of $1000 \mathrm{~W} / \mathrm{m}^{2}$;

FS, Security Factor (1.2);

Pmed, Annual Medium Power of the electric load (in kW/year);

FP, Performance Factor (in \%);

$N P$, Number of identical elements of photovoltaic subsystem (photovoltaic panels).

The Used Annual Energy for PV subsystem, $U A E \_P V$, (in $\mathrm{kWh}$ ) is:

$$
U A E_{-} P V=P V \% \cdot P \text { med } \cdot 8760
$$

The Produced Annual Energy for PV subsystem, $P A E \_P V$, (in kWh) (considering that $F P$ is the relation between the electric energy consumed by the electric load and the energy that the subsystem can produce) is:

$$
P A E_{-} P V=\frac{F S \cdot U A E_{-} P V}{F P}
$$

The equivalent Annual Hours of Use, $A H U$, (in hours), are obtained from the annually available irradiation in each point and the irradiation standard conditions, according to:

$$
A H U=\frac{G_{A} \cdot 365}{G_{0}}
$$

Each panel will have a capacity $P P \_P V$, (in $\mathrm{kW}$ ) of:

$$
P P_{-} P V=\frac{P A E_{-} P V}{N P \cdot A H U}
$$


The costs of equipment for PV panels, $C P V$, are associated to their nominal power, $P P \_P V$, and the computational tools have "lookup tables" to select those costs. Since $G_{A}$ is a geographic data type that has a single value on every location (it is better when the location is free of shadows and exposed southward), $A H U$ and $P P \_P V$ (and therefore, $C P V$ ) have also a value assigned for each cell (location) of the geographic grid of the study area.

If the user of software selects a power capacity, $Q$, (in $\mathrm{kW}$ ) for the hybrid system, the Produced Annual Energy, $P A E \_P V$, will be then:

$$
P A E_{-} P V=P V \% \cdot Q \cdot A H U
$$

And each panel has a capacity $P P \_P V$, of:

$$
P P_{-} P V=\frac{P A E_{-} P V}{N P \cdot A H U}=\frac{P V \% \cdot Q}{N P}
$$

The size (PHPV) of the photovoltaic subsystem of the hybrid system is:

$$
P H P V=N P \cdot P H_{-} P V
$$

The rest of the equipment can be sized following similar reasoning, with specific equations and "lookup tables" obtaining the size PHW and PHG of the wind and diesel generator subsystems, respectively; then, the rated power of the hybrid system, $\mathrm{PH}$, is:

$$
P H=P H P V+P H W+P H G
$$

With the rated power of the hybrid system we can obtain the costs of the components, like batteries or inverters, associated with the equipment of the whole hybrid system.

The Levelized Energy Costs needs the evaluation of each kind of costs; for every point of the geographical area studied, the costs associated with the equipment comprising the hybrid system (CEqu) are:

$$
C E q u=C G+C W+C P V+C B a t+C I n v
$$

where:

$C G$ are the equipment related costs of the electric generating unit, distributed over its entire useful life, using the discount rate and the unit's years of useful life, i.e. obtaining annual costs, in $€ /$ year.

$C W$ are the equipment-related costs of the wind turbines in $€ /$ year.

$C P V$ are the equipment-related costs of the photovoltaic solar panels, in $€ /$ year.

CBat are the equipment-related costs of the energy storage systems (batteries), in $€$ /year.
CInv are the equipment-related costs of other components (inverters if necessary, protection devices), in €/year.

Furthermore, costs associated with the installation of the whole hybrid system (in every point of the studied area) are evaluated, as well as the annual operation and maintenance costs (CHS), which are:

$$
C H S=P H \cdot C O M+C I n s
$$

where:

$\mathrm{PH}$ is the rated power of the hybrid system, in $\mathrm{kW}$.

COM are the annual costs per installed $\mathrm{kW}$, associated with the annual operation and maintenance costs of the hybrid system, in $€ / \mathrm{kW}$ and year.

CIns are the costs associated with the installation of the hybrid system, distributed over its entire useful life, in $€$ /year.

Then, the LEC of the isolated hybrid system, $L E C_{0}$, for every point of the geographical area of study are:

$$
L E C_{0}=\frac{C E q u \cdot\left(1-\frac{S E}{100}\right)+C C o m b+C E P+C H S}{A E P}
$$

where:

$S E$ is the percentage of economic subsidy awarded for the equipment.

CComb is the annual cost to fuel, in every site, the electric generating unit subsystem, in $€ /$ year. $C E P$ is the annual cost associated with the pollutant emitted by the electric generating unit subsystem (subject to possible "environmental taxation”), in €/year.

$A E P$ is the annual energy produced by the hybrid system, in $\mathrm{kWh} /$ year.

2) Connected hybrid distributed generation systems. In addition to the initial data already used for isolated hybrid systems, a geographical data grid with the connection costs to electric power distribution networks was used for connected hybrid systems (CCEN, in $€ / \mathrm{kW}$ and year); this was obtained according to the power capacity of the hybrid system.

The price of electric energy ( $P E E, € / \mathrm{kWh}$ ) was introduced in the formulation of the LEC, in addition to the abovementioned costs, that corresponds to the following values:

$$
\begin{aligned}
& P E E=P E H S \Leftrightarrow A E P \geq U A E \\
& P E E=P D U \Leftrightarrow A E P<U A E
\end{aligned}
$$

where: 
PEHS is the fixed price of renewable energy on sale (price regulated by the Spanish Government, in order to promote the integration of distributed resources in the power system; this regulated price also depends on $\mathrm{PH}$ ). This price is used when produced electric energy is greater than consumed electric energy, and the rest can be sold.

$P D U$ is the price of the electric energy purchased from distribution utilities. The two components of the $L E C_{1}$ in connected hybrid systems were:

$$
\begin{gathered}
C O S T=L E C_{0} \cdot A E P+P H \cdot C C E N \\
P R I C E=P E E \cdot(U A E-A E P)
\end{gathered}
$$

Then:

$$
L E C_{1}=\frac{C O S T+P R I C E}{A E P}
$$

where:

$L E C_{0} \cdot A E P$ in (14) substitutes the expression of the numerator in the previous equation (11), in $€ /$ year.

$A E P$ is the annual energy produced by the hybrid system, in $\mathrm{kWh} /$ year.

$U A E$ is the consumed annual energy, supplied by the hybrid system, in $\mathrm{kWh} /$ year.

$\mathrm{PH}$ is the rated power of the hybrid system, in $\mathrm{kW}$.

CCEN are the connection costs to electric power distribution networks of the hybrid system, in $€ / \mathrm{kW}$ and year; these costs depend on the geographical characteristics of the zone, on the existing electric power distribution networks and on the cost associated with the equipment required to make the connection.

If negative $L E C_{1}$ values are obtained, it means that the costs per $\mathrm{kWh}$ are smaller than the profits obtained from the sale of energy surpluses. Hence, a scenario with connection to the power distribution network can provide useful information about the resultant profits that the hybrid system can generate in each studied location, classified from large to small profits (from small to large costs, when $L E C_{1}$ is lower than zero). This information can be very useful for users, utilities and administrative authorities.

\section{Computational Results}

Extensive computational results were obtained throughout the region of La Rioja (Spain), with the result of the Levelized Energy Costs (LEC) obtained for an isolated hybrid system. In this study, the hybrid system consisted on:
- a photovoltaic subsystem (with a weight of 30\%) formed by photovoltaic solar panels that can be grouped in a range from 1 to $20 \mathrm{kWp}$ and 30 years of useful life;

- and a "biodiesel" subsystem, (with the remaining $70 \%$ ) consisting of a selected electric generating unit chosen with ranges from 5 to $20 \mathrm{~kW}, 30$ years of useful life, 23\% efficiency.

The hybrid system was designed so that, in every point of the zone studied (a square pattern called a "grid-cell", measuring 100x100 meters, with a total of 501,725 gridcells in the area of study), it could supply an uninterrupted electrical load with $12 \mathrm{~kW}$ peak power, $70 \%$ utilization factor and a daily consumption cycle of 24 hours. The results corresponded to residential or small agricultural-industrial isolated demands.

The economic scenario did not consider any economic subsidy for the equipment or for environmental revenues (for avoiding the emission of atmospheric pollutants), though all the selected subsystems provided "green energy”.

The hybrid system was referred to isolated systems. In addition, it was resized in power capacity to meet demand. For this reason, batteries had to be incorporated as an electric energy storage system with an energy reserve of 24 hours and with other components (inverters) to allow DC-AC conversion.

We obtained the solar photovoltaic resources for the entire region of La Rioja and related costs, using the computational tools mentioned in [1] and [8] (in the form of a geographical information surface or grid).

The computational tools for spatial analysis were used to build the grid of resources and fuel availability for the selected electric generating units (for a specific energy of bio-diesel oil, in our case-study, 37,500 MJ/ton). In this grid, the geographical cost of distributing the bio-fuel for the studied area in each study point was added to the cost of one litre of bio-diesel oil at the available "green" petrol stations $(0.95 € / \mathrm{l})$. Then, a distributed cost of biofuel was obtained, where the highest value corresponded to the locations farthest away from the "green" petrol stations and from transport infrastructures (roads).

Different types of results were obtained from the study; some were single values that were the same for all the locations in the studied area, such as the size of the energy storage subsystems (batteries), the size of other components (inverters) or the size of each selected electric generating unit in the scenario studied. Other results were obtained in a grid format, such as $L E C_{0}$, or the cost of produced electric energy (one different value in every point).

In the scenario studied, the batteries were resized to 108 $\mathrm{kWh}$ of capacity to provide a 24-hour reserve; the inverters were resized to $14.4 \mathrm{~kW}$ and the bio-diesel electric generating unit had a rated power of $5 \mathrm{~kW}$. 


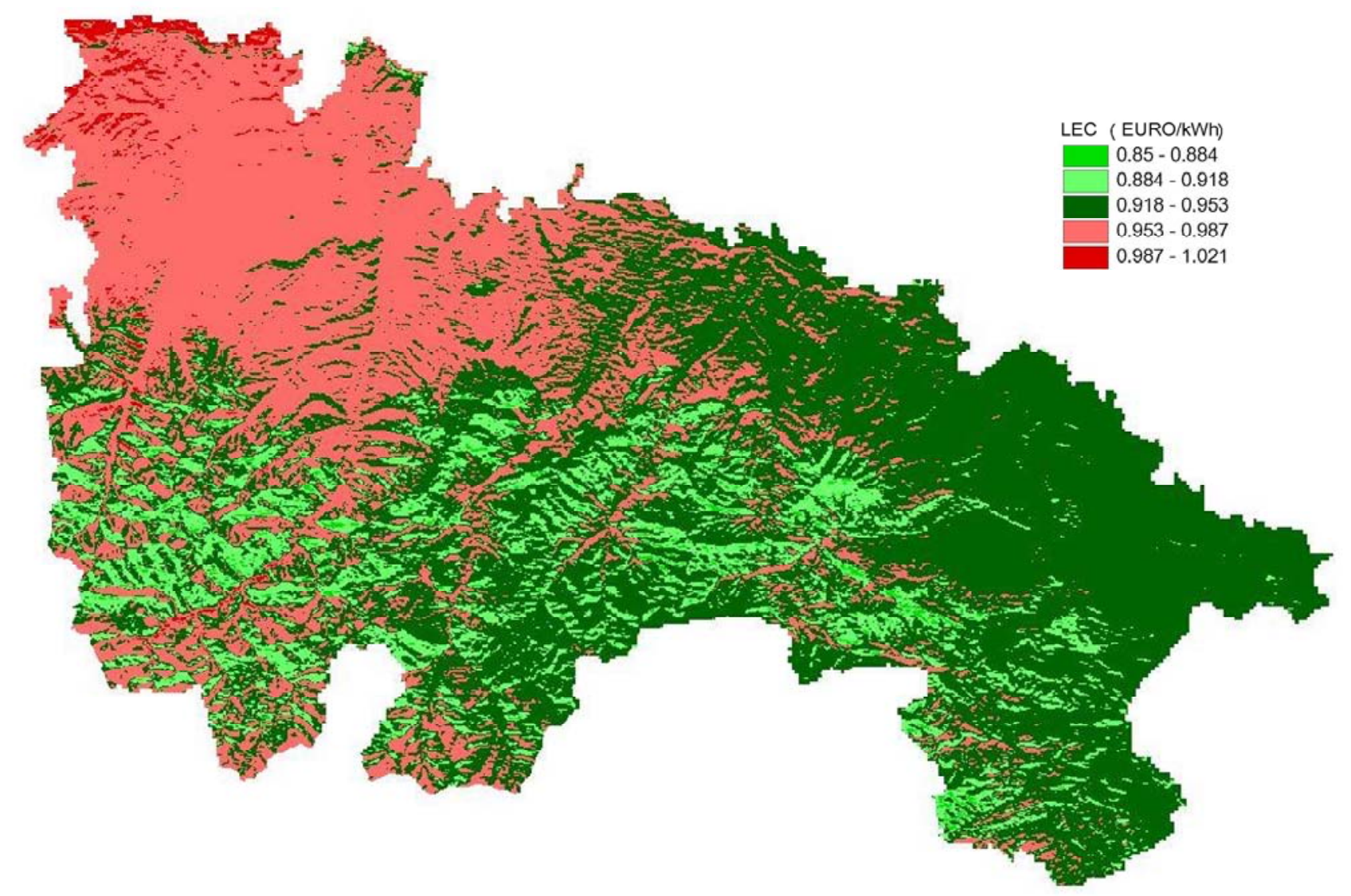

Fig. 1. LEC for an isolated hybrid system in La Rioja (Spain).

Fig. 1 shows the results obtained for the whole region in study: La Rioja. The grid (for an isolated hybrid system) specifically indicates the $L E C_{0}$ : the most economic locations were those with the lowest $L E C_{0}$ values (bright green colour); this indicated the best locations for installing the hybrid system to meet demand, taking into account the quantity and quality of the available energy resources. The great irradiation in the Southeast, or the sunny South-oriented hillsides everywhere configure the best places.

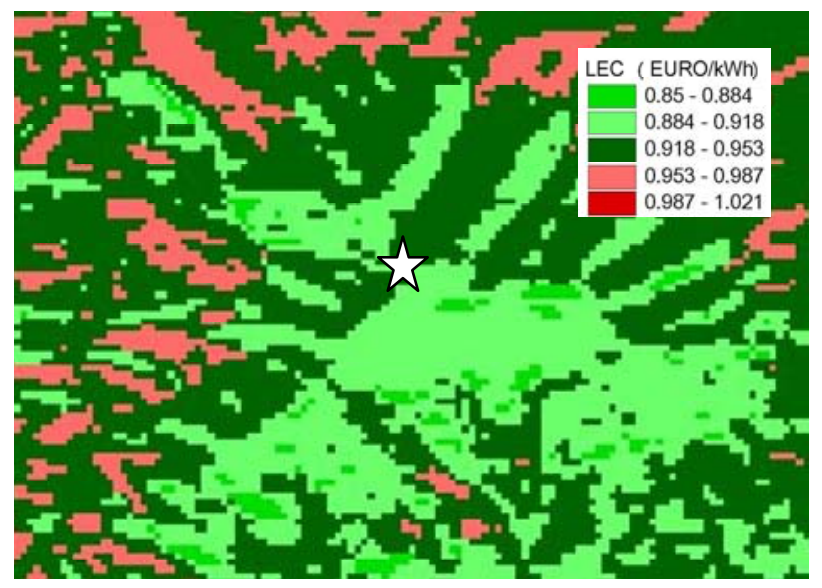

Fig. 2. Zoom of Fig. 1 in the surroundings of Cabi Monteros Peak (the white star in the middle).
Note that the South-oriented hillsides of the mountains (bright green colour) are ideal locations from an economic standpoint, and the interiors of valleys and North-oriented hillsides of the mountains (red colour) are the worst zones. These ideal locations are the best points (in the studied scenario) due to the high value of solar energy resources in those locations allowing ample power supply to cover the electric load mainly from the wind energy.

In these locations, solar resources on sunny hillsides facing south displayed higher intensity and quality, and the generation of electric energy (requested by demand) is more economic (since the equipment could be resized to a lower rated installed power and the utilization hours of the diesel subsystem are lower than in other locations with poorer solar resources).

Fig. 2 shows the surroundings of the best location in the studied area: the South-oriented hillsides of Cabi Monteros Peak, in the proximities of the village of Arnedillo. Note the grid-cell structure.

In the scenario studied here, the cost per unit of produced energy had a minimal value of $0.850 € / \mathrm{kWh}$. In the deep valleys, where the diesel electric generating unit subsystem had to work more hours to supply the electric energy that the sun cannot provide in these areas, the cost was $1.021 € / \mathrm{kWh}$ (in the worst locations). Although these $€ / \mathrm{kW}$ values calculated at several locations would seem 
to be high, they are actually significantly lower than the final costs of electrical power supply (in these locations) associated with the electric power line (that has to be built as part of the expansion of the power network) and the corresponding equipment required to meet the demand in a similar connected scenario.

\section{Conclusions}

This paper has presented suitable models for calculating the Levelized Energy Costs of hybrid distributed generation facilities. The models were implemented to develop software tools and applied in the region of La Rioja (Spain), providing a series of very useful geographical and technical results for decision-makers in power distribution systems planning.

The software and the created models can be used to analyse electricity production costs for a large set of alternatives of mixed renewable and non-renewable resources from data for a given geographical area; the software and models can evaluate both isolated distribution generation and distributed generation connected to the existing electric power distribution networks.

These models consider the costs of the necessary equipment in order to size the hybrid distributed generation system, as well as economic factors (annuity factors, subsidy for purchasing equipment, tax incentives, tax and duty exemptions, price regulated for electric energy on sale, etc.), and geographical factors (fixed and variable geographical installation costs, installation operation and maintenance costs, geographical distributed refuelling costs, etc.).

Using combinations of these parameters, carried out by the user of the developed software tool, a huge range of distributed generation alternatives can be studied and the different Levelized Energy Costs can be analyzed according to existing resources, different energy subsidy polices, the electricity market prices, or electric load to be supplied (sizing of the hybrid system), always taking into account the characteristics of the geographically distributed resources and the characteristics of power demand.

\section{Acknowledgement}

The authors would like to thank the "Ministerio de Ciencia e Innovación” of the Spanish Government for supporting this research under the Project ENE200914582-C02-02, as well as we thank the European Union for its support by the ERDF (European Regional Development Fund).

\section{References}

[1] I.J. Ramírez-Rosado, L.A. Fernández-Jiménez, C. Monteiro, V. Miranda, E. García-Garrido and P.J. Zorzano-Santamaría, "Powerful Planning Tools: GIS power up Distributed Generation”, in IEEE Power \& Energy Magazine, March/April 2005, Vol. 3(2), pp. 56-63.

[2] B. Ai, H. Yang, H. Shen, X. Liao, "Computer-aided design of PV/wind hybrid system”, in Renewable Energy, Vol. 28(10), 2003, pp. 1491-1512.

[3] M.A. Elhadidy, "Performance evaluation of hybrid (wind/ solar/diesel) power systems”, in Renewable Energy, Vol. 26(3), 2002, pp. 401-413.

[4] A.L. Schmid, C.A.A. Hoffmann, "Replacing diesel by solar in the Amazon: short-term economic feasibility of PV-diesel hybrid systems”, in Energy Policy, Vol. 32(7), 2004, pp. 881898.

[5] P. Nema, R.K. Nema, S. Rangnekar, “A current and future state of art development of hybrid energy system using wind and PV-solar: A review”, in Renewable and Sustainable Energy Reviews, Vol. 13, 2009, pp. 2096-2103.

[6] J. McKoy, K. Johnston, Using ArcGIS Spatial Analyst, Environmental Systems Research Institute Inc. Editor (ESRI), Redlands CA, (2001).

[7] K. Scharmer, J. Greif, coordinators, The European Solar Radiation Atlas, École des Mines de Paris, France, (2000).

[8] C. Monteiro, I.J. Ramírez-Rosado, et al., "Spatial Analysis Tool to Evaluate Spatial Incremental Costs on Electric Distribution”, in Power Tech Proceedings, IEEE Porto, vol. 1, 4 pages. D.O.I.:10.1109/PTC.2001.964583, (2001). 